\title{
XMM-Newton X-ray spectroscopy of classical T Tauri stars
}

\author{
J. Robrade and J. H. M. M. Schmitt
}

Hamburger Sternwarte, Universität Hamburg, Gojenbergsweg 112, 21029 Hamburg, Germany

e-mail: jrobrade@hs.uni-hamburg.de

Received 23 September 2005 / Accepted 13 December 2005

\begin{abstract}
We present results from a comparative study of XMM-Newton observations of four classical T Tauri stars (CTTS), namely BP Tau, CR Cha, SU Aur and TW Hya. In these objects coronal, i.e. magnetic, activity and as recently shown, magnetically funneled accretion are the processes likely to be responsible for the generation of X-ray emission. Variable X-ray emission with luminosities in the order of $10^{30} \mathrm{erg} / \mathrm{s}$ is observed for all targets. We investigate light curves as well as medium and high-resolution X-ray spectra to determine the plasma properties of the sample CTTS and to study the origin of their X-ray emission and its variability. The emission measure distributions and observed temperatures differ significantly and the targets are dominated either by plasma at high densities as produced by accretion shocks or by predominantly hotter plasma of coronal origin. Likewise the variability of the X-ray luminosity is found to be generated by both mechanisms. Cool plasma at high densities is found in all stars with detected O VII triplet emission, prevented only for SU Aur due to strong absorption. A general trend is present in the abundance pattern, with neon being at solar value or enhanced while oxygen, iron and most other metals are depleted, pointing to the presence of the inverse FIP effect in active coronae and possibly grain formation in evolved disks. We find that both accretion shocks and coronal activity contribute to the observed X-ray emission of the targets. While coronal activity is the dominant source of X-ray activity in the majority of the CTTS, the fraction for each process differs significantly between the individual objects.
\end{abstract}

Key words. stars: activity - stars: coronae - stars: late-type - stars: pre-main sequence - X-rays: stars

\section{Introduction}

Young, late-type pre main-sequence stars, known as T Tauri stars, are copiously found in or near star forming regions. Historically, they are classified according to their $\mathrm{H} \alpha$ equivalent width as classical T Tauri stars (CTTS) with $E W>10 \AA$ or otherwise as weak line T Tauri stars (WTTS). The principal physical difference between these two classes is that CTTS are thought to be in an earlier evolutionary stage, i.e. they still possess a disk containing dust and gas and are accreting matter. WTTS are thought to be more evolved, to have mainly lost their disks and are approaching the main-sequence. While CTTS are generally younger with ages of a few up to $\sim 10 \mathrm{Myr}$ and the overall fraction of CTTS in a given star-forming region decreases with age, both types of T Tauri stars are commonly found in the same star-forming regions, indicating individual evolutionary time scales. Since the $\mathrm{H} \alpha$-line is often time-variable, a classification solely based on its strength is sometimes misleading and other or additional spectral features have been used to identify and classify CTTS more reliably. The existence of a disk containing a significant amount of matter and ongoing accretion onto the host star impose major differences between the two types of TTS, leading to different spectral properties which can be observed at different wavelengths. The effects of the warm and probably structured disk are for instance reflected in the different infrared designations of CTTS (Class II) and WTTS (Class III) due to their additional near-infrared emission, which is an important indicator for CTTS. Consequently, the different criteria and underlying data used for the identification of CTTS may introduce selection effect in the respective samples.

Both types of T Tauri stars are well known strong and variable X-ray emitters and large numbers of these objects were detected with Einstein (Feigelson \& DeCampli 1981; Feigelson \& Kriss 1989) and ROSAT (Feigelson et al. 1993; Neuhäuser et al. 1995). Their X-ray emission and the observed flaring was usually interpreted as a scaled-up version of coronal activity in analogy to cool main-sequence stars. This picture is still valid and the X-ray emission from WTTS is thought to originate from coronal activity. However, T Tauri stars are thought to be at least in their early stages fully convective, therefore the question arises about the underlying processes for the generation of the magnetic field and the influence of accretion on the stellar structure and corona. Further on, the evolutionary stages of young stars and their high energy emission is of major importance for the process of planetary formation via its effects on chemistry, dust and protoplanets. A comprehensive review of high energy processes and underlying physics in young stellar objects, summarising the results of the era prior to Chandra 
and XMM-Newton, is presented by Feigelson \& Montmerle (1999).

The largest sample of pre main-sequence stars are found in the Orion Nebula Cloud. Using Chandra ACIS observations, Feigelson et al. (2003) analysed a sample of 500 PMS stars with known basic properties. An even deeper exposure, named the Chandra Orion Ultradeep Project (COUP) was presented recently, see e.g. Preibisch et al. (2005), confirming and extending their results. The X-ray luminosity of CTTS in this sample was found to be correlated with bolometric luminosity. No strong correlation between $L_{X}$ and rotation as observed for main-sequence stars was found, contrary, $L_{X}$ correlates with $L_{\text {bol }}$ and stellar mass, indicating the presence of different, e.g. turbulent, dynamos or of supersaturation effects in solartype dynamos. The question, whether the presence of an accretion disk influences the stellar X-ray emission is still under debate. While Feigelson et al. (2003) found no intrinsic differences of X-ray activity related to the presence of a circumstellar disk, the results found by Preibisch et al. (2005) indicate that accretion diminishes $L_{X}$ on average and weaken the $L_{\mathrm{X}} / L_{\text {bol }}$ correlation. A similar influence was found by Stelzer $\&$ Neuhäuser (2001) in the X-ray properties of young stars in the Taurus-Auriga complex observed with ROSAT PSPC. The COUP sources are rather consistent with the bulk of their X-ray emission being produced in typical coronae. In general, magnetic activity in CTTS may also involve the circumstellar material, e.g. via star-disk interaction, but the topology of their magnetic fields in virtually unknown; Favata et al. (2005) present evidence for magnetic star-disk interaction in the analysis of larger flares on several COUP objects.

Moreover, recent high-resolution spectroscopy of CTTS with Chandra and XMM-Newton, indicated accretion shocks at least as an additional mechanism for the generation of X-ray emission in CTTS. In this interpretation X-ray emission is generated by a magnetically funneled accretion stream falling onto small areas of the stellar surface, producing shocked high density plasma (Shu et al. 1994; Calvet \& Gullbring 1998). Since in magnetospheric accretion the matter falls from several stellar radii at nearly free-fall velocity, strong shocks and consequently hot plasma emitting in the X-ray regime are formed. This contribution is expected to be at energies below $0.5 \mathrm{keV}$ and is distinguishable from cool coronal plasma by its density; therefore it can be recognised only through high resolution X-ray spectroscopy. It is thus not contradictory that the COUP sample based on ACIS data with medium resolution spectroscopy and poorer sensitivity at low energies finds accretion to play no significant role.

TW Hya was the first example of a CTTS, where observational evidence for an accretion scenario could be found in $\mathrm{X}$-ray data. Analysis using density sensitive lines of He-like triplets (e.g. O VII, Ne IX) indicated very high densities, exceeding by far the densities previously found in coronal plasma from any other star (Kastner et al. 2002; Stelzer \& Schmitt 2004). These low line ratios were also found in the spectra of BP Tau (Schmitt et al. 2005), again strongly supporting an accretion scenario. However, accretion is only capable to produce plasma with temperatures of a few MK and therefore contributes nearly exclusively to the low energy X-ray emission.
The observed flaring and additional hard emission attributed to plasma with temperatures of several tens of MK in BP Tau and other CTTS require the presence of an additional X-ray generating mechanism such as magnetic activity. In this work we use the term "coronal activity", but more complex phenomena like star-disk interactions cannot be ruled out. While TW Hya like objects where accretion is thought to be the dominant source of the X-ray emission are probably rare, the BP Tau observation revealed that accretion as an additional X-ray generating mechanism may be more common. Another X-ray generating mechanism, shocks in strong Herbig-Haro outflows that may also produce a soft X-ray excess, was proposed for jet-driving CTTS (which resemble Class I protostars) like DG Tau (Güdel et al. 2005). We note that this model cannot explain the soft excess in the case of TW Hya or the other sample CTTS.

We utilise XMM-Newton data from four X-ray bright CTTS to study the origin of their X-ray emission in accretion shocks vs. coronal activity. We specifically use medium and high resolution X-ray spectra and spectrally resolved X-ray light curves to investigate the physical properties of our target stars BP Tau, CR Cha, SU Aur and TW Hya and to check the relevance of these mechanisms for the generation of the X-ray emission and variability in these young stars. This work is the first comparative X-ray study of CTTS using high and medium resolution spectra so far, therefore it is complementary to large sample studies of a specific star forming region such as COUP. Moreover, the data of CR Cha and partly of SU Aur and BP Tau is analysed and presented here for the first time. Results derived from RGS spectra of BP Tau and the simultaneous obtained UV data were presented in our previous letter (Schmitt et al. 2005), while the data on TW Hya first presented by Stelzer \& Schmitt (2004) is re-analysed for purposes of comparison in a manner identical to the analysis of all other sources. The plan of our paper is as follows: in Sect. 2 we describe the individual targets and previous X-ray results, in Sect. 3 the observations and the methods used for data analysis and in Sect. 4 we present and discuss the results subdivided into different physical topics, followed by a summary and our conclusions in Sect. 5 .

\section{The CTTS sample}

BP Tau is a CTTS with spectral type K5-K7 associated with the Taurus-Auriga star forming region at a distance of $140 \mathrm{pc}$ as discussed by Wichmann et al. (1998). Optical and infrared measurements clearly show the typical characteristics of excess emission produced by accretion (Gullbring et al. 1998). BP Tau has a moderate inclination angle of roughly $30^{\circ}$ and its disk was found to be quite compact (Muzerolle et al. 2003). X-ray emission from BP Tau with $L_{\mathrm{X}} \sim 10^{30} \mathrm{erg} / \mathrm{s}$ was already measured with the Einstein Observatory (Walter \& Kuhi 1981), the RASS luminosity was determined as $0.7 \times 10^{30} \mathrm{erg} / \mathrm{s}$ (Neuhäuser et al. 1995). Gullbring et al. (1997) presented an analysis of simultaneous optical and ROSAT observations of BP Tau. Finding no correlation between optical and X-ray variability they concluded that the optical emission is attributed to accretion while the X-ray emission arises from magnetically active regions. Initial results of our XMM-Newton observation of BP Tau with special focus on the RGS data and density 
diagnostics with the He-like triplet of O VII were presented in our previous letter (Schmitt et al. 2005), where evidence for high density plasma and/or intense UV radiation is presented, supporting the presence of an accretion funnel in BP Tau and a rough estimate of BP Tau's mass accretion rate as derived from simultaneous UV measurements was given.

CR Cha with spectral type $\mathrm{K} 2$ is located in the Chamaeleon I cloud, a star forming region at a distance of $140-150 \mathrm{pc}$ with a measured RASS luminosity of $1.5 \times$ $10^{30} \mathrm{erg} / \mathrm{s}$ (Feigelson et al. 1993). It was classified as CTTS via $\mathrm{H} \alpha$, see e.g. Reipurth et al. (1996), and according to Meeus et al. (2003) it is dominated by small amorphous silicates, indicating large amounts of unprocessed dust. The archival XMM-Newton data of CR Cha is analysed and presented here for the first time.

SU Aur is classified as G2 subgiant and also a member of the Taurus-Auriga star forming region, therefore we adopt a distance of $140 \mathrm{pc}$. For this very luminous and variable object also a wider range of values of its optical properties is given in literature, see e.g. DeWarf et al. (2003). It was sometimes classified as WTTS, but a clear IR-excess confirms its status as a CTTS, viewed nearly edge on, with a rather high accretion luminosity (Muzerolle et al. 2003), thus causing the strongest absorption signatures in our sample. SU Aur is one of the brightest known CTTS, its strong X-ray emission was already detected with the Einstein Observatory (Feigelson \& DeCampli 1981) with an X-ray luminosity of $3.0 \times 10^{30} \mathrm{erg} / \mathrm{s}$. A similar value $\left(3.7 \times 10^{30} \mathrm{erg} / \mathrm{s}\right)$ was derived from RASS data by Neuhäuser et al. (1995), making it the brightest target in our sample, especially when considering the unabsorbed X-ray luminosity. Some results of the XMM-Newton observation are presented in an analysis of star forming regions by Pallavicini et al. (2004), who showed that SU Aur is both extremely hot and variable.

TW Hya is classified as a K7-K8 star and is the nearest object in our sample. At a distance of only $56 \mathrm{pc}$ it is located in the TW Hydra association, a nearby young but diffuse stellar associations, see e.g. Zuckerman et al. (2001). TW Hya is viewed nearly pole on and due to its proximity and lack of obscuring clouds it can well studied at all wavelengths. With an estimated age of nearly $10 \mathrm{Myr}$ it is also one of the oldest known stars, still accreting matter. Kastner et al. (1999) analysed ASCA and ROSAT data of TW Hya and found that a model with plasma temperatures of $\sim 1.7$ and $\sim 9.7 \mathrm{MK}$ and an X-ray luminosity of $\sim 2 \times 10^{30} \mathrm{erg} / \mathrm{s} \mathrm{describes}$ both observations well. TW Hya was also observed with the Chandra HETGS detector (Kastner et al. 2002), providing high resolution spectra, which were modelled with an iron depleted and neon enhanced plasma and a emission measure distribution showing a sharp peak around $3 \mathrm{MK}$. The analysis of density sensitive lines indicated plasma at high densities $\left(\log n_{\mathrm{e}}=13\right)$ and the inferred plasma properties were found to be consistent with the X-ray emission to be generated by funneled mass accretion from the circumstellar disk. Several moderate "flares" were detected on TW Hya during the ASCA and Chandra observation; however, the untypical shape of the flare light curve and the seemingly constant spectral properties led Kastner et al. (2002) to conclude that most or all of the X-ray emission of TW Hya originates from accretion.
Table 1. Basic properties for our sample CTTS. Spectral types taken from Simbad database, distances adopted from Wichmann et al. (1998), ages from Gullbring et al. (1998): BP Tau, Natta et al. (2000): CR Cha, DeWarf et al. (2003): SU Aur, Makarov \& Fabricius (2001): TW Hya, $L_{x}$ are ROSAT values $(<2.4 \mathrm{keV})$.

\begin{tabular}{lcccc}
\hline \hline Target & Spec.T. & Dist.(pc) & Age $(\mathrm{yr})$ & $L_{x}\left(10^{30} \mathrm{erg} \mathrm{s}^{-1}\right)$ \\
\hline BP Tau & K5V & 140 & $6 \times 10^{5}$ & 0.7 \\
CR Cha & K2 & 145 & $1 \times 10^{6}$ & 1.5 \\
SU Aur & G2III & 140 & $4 \times 10^{6}$ & 3.7 \\
TW Hya & K8V & 56 & $8 \times 10^{6}$ & 2.0 \\
\hline
\end{tabular}

Table 2. Observation log of our sample CTTS, duration of prime instrument/RGS(filtered).

\begin{tabular}{lccc}
\hline \hline Target & Obs.Mode & Obs. Time & Dur. (ks) \\
\hline BP Tau & FF/thick & 2004-08-15T06:14-16T18:51 & $132 / 124$ \\
CR Cha & FF,LW/med. & 2001-02-24T05:04-15:49 & $39 / 72$ \\
SU Aur & FF/thick & 2001-09-21T01:27-22T14:18 & $130 / 126$ \\
TW Hya & FF/med. & 2001-07-09T05:51-14:01 & $30 / 28$ \\
\hline
\end{tabular}

Results of the XMM-Newton observation of TW Hya obtained with a somewhat different analysis were presented by Stelzer \& Schmitt (2004), who found that the XMM-Newton data can be well explained by X-ray emission from a metal depleted accretion shock with an X-ray luminosity of $\sim 1.5 \times 10^{30} \mathrm{erg} / \mathrm{s}$, consistent with the Chandra results. Different conclusions were derived at on the interdependent properties mass accretion rate and surface area of the shock region, which is assumed to fill either below one percent or up to a few percent of the stellar surface. Here the TW Hya data is independently re-analysed to ensure consistency and allow a comparison of our results.

\section{Observation and data analysis}

All sample CTTS were observed with XMM-Newton using somewhat different detector setups with exposure times in the range of 30-130 ks. Data were taken with all X-ray detectors, which were operated simultaneously onboard XMM-Newton, respectively the EPIC (European Photon Imaging Camera), consisting of the MOS and PN detectors and the RGS (Reflection Grating Spectrometer). Note that for SU Aur no PN data is available and different filters were used, the thick filter for the BP Tau and SU Aur and the medium filter for the CR Cha and TW Hya observations. Further on, the signal to noise ratio differs for the various targets and instruments. For CR Cha only a fraction of the original data from the additionally split observation could be used for analysis because of very high background contamination; the RGS data from two exposures was merged with the tool "rgscombine" provided with SAS 6.5, but the RGS spectrum of CR Cha is still underexposed. The EPIC data is quality is sufficient for all targets. In addition, strong absorption affects the data quality especially at lower energies. The details of our observations and used data are described in Table 2, a detailed description of the XMM-Newton instruments can be found in Ehle et al. (2003). 
Data analysis was performed with the XMM-Newton Science Analysis System (SAS) software, version 6.0. Images, light curves and spectra were produced with standard SAS tools and standard selection criteria were applied for filtering the data, see Loiseau et al. (2004). X-ray spectral analysis was carried out with XSPEC V11.3 (Arnaud 1996), while for line fitting purposes we used the CORA program (Ness \& Wichmann 2002), assuming Lorentzian line shapes. Individual line fits are used to investigate the density sensitive forbidden and intercombination lines of He-like triplets. Line counts are derived keeping the line spacing fixed within a triplet and using an overall line width for all lines. Spectral analysis of EPIC data is performed in the energy band between 0.3-10.0 keV, while the RGS first order spectra in the full energy range, i.e. $0.35-2.5 \mathrm{keV}(5-35 \AA)$, are used whenever data quality permits. While the RGS obviously has the highest spectral resolution, the EPIC detectors are more sensitive and are able to measure higher energy X-rays; the MOS detectors provide a slightly better spectral and spatial resolution, the PN detector is more sensitive. We emphasize that the data were analysed simultaneously but not co-added, thus providing sufficient signal for all observations and ensuring a consistent analysis for all targets. All periods affected by proton flares were removed from spectral analysis and for TW Hya the PN data was cleaned for some moderate pile-up. The background was taken from source free regions on the detectors.

For the analysis of the X-ray spectra we use multitemperature models with variable but tied abundances, i.e. the abundance pattern was assumed to be the same in all temperature components. Such models assume the emission spectrum of a collisionally-ionized optically-thin gas as calculated with the APEC code, see e.g. Smith et al. (2001). Since the RGS is not very sensitive above $2.0 \mathrm{keV}$, we use EPIC data to determine the properties of plasma with temperatures above 20 MK. In order to account for calibration uncertainties of the different detectors, see e.g. Kirsch et al. (2004), the normalization between instruments was taken as a free parameter for each type of instrument, i.e. for MOS, PN, RGS. Our fit procedure is based on $\chi^{2}$ minimization, therefore spectra are always rebinned to satisfy the statistical demand of a minimum value of 15 counts per spectral bin. All errors are statistical errors given by their $90 \%$ confidence range and were calculated separately for abundances and temperatures by allowing variations of normalizations and respective model parameters. Note that additional uncertainties arise from uncertainties in the atomic data and instrumental calibration which are not explicitly accounted for.

The applied models use three temperature components, models with additional temperature components were checked, but did not improve the fit results significantly. Since the APEC models do not account for high density plasma, resolved density sensitive lines are excluded from the global fits for TW Hya. Abundances are calculated relative to solar photospheric values as given by Anders \& Grevesse (1989). For iron and oxygen we use the updated values of Grevesse \& Sauval (1998). Application of the new but controversial solar abundances published by Asplund (2005) would further increase the neon abundance in our sample stars compared to other metals, but not change our conclusions significantly. We note that relative results between the CTTS are not affected by the underlying solar abundance pattern. For elements with overall low abundances and no significant features in the X-ray spectra, i.e. $\mathrm{Al}, \mathrm{Ca}, \mathrm{Ni}$, the abundances were tied to the iron abundance. When data quality permits, we determine the abundances of individual elements, global abundances are used if no features are present in the spectra. This is for instance the case for targets with strong absorption, where e.g. the stronger carbon lines at the low energy end of the detectors are completely absorbed. We simultaneously modelled the temperatures and emission measures $\left(E M=\int n_{\mathrm{e}} n_{\mathrm{H}} \mathrm{d} V\right)$ of the components and checked the derived results for consistency. X-ray luminosities were then calculated from the resulting best fit models.

Absorption in the circumstellar environment and possibly also in the interstellar medium is significant for CTTS and is applied in our modelling. Since absorption is supposed to be slightly variable and, in addition, optical and IR measurements often give a wider range of values, it is kept as a free parameter in our analysis. In general, the derived fit results are quite stable, but note that some of the fit parameters are mutually dependent, thus affecting especially absolute values. Interdependence mainly affects the low energy region of the spectra, where the strength of absorption, the emission measure and abundances of elements with emission lines in the respective temperature range strongly depend on each other. While some effects can be prevented by the methods described above, models with different absolute values of the mentioned parameters but only marginal differences in its statistical quality may be fitted to the data, however ratios and relative changes of these properties are again very robust.

\section{Results}

\subsection{X-ray light curves}

To study time variability and its origin we first investigated the X-ray light curves of our sample CTTS. In Fig. 1 we show background subtracted light curves with a temporal binning of $1000 \mathrm{~s}$, extracted from a 50" radius circular region around each source and cleaned for obvious data dropouts.

A flare with a rise in count rate of factor 2.5-3 occurred during the BP Tau observation and a smaller flare (factor 1.5) during the CR Cha observation. In the SU Aur data several flares were detected, the largest one showing an increase in X-ray brightness by a factor of 3. TW Hya does not exhibit a flare during the XMM-Newton observation, any variability remains in the range of only $10 \%$. However, light curve variations with factors around two are also known for TW Hya (Kastner et al. 1999, 2002), who noted three "flares" during $140 \mathrm{ks}$ (ASCA, $94 \mathrm{ks}+$ Chandra, $48 \mathrm{ks}$ ) observation time but no spectral changes were detected during the periods of increased $\mathrm{X}$-ray brightness. The average time scale for flares on CTTS appears to be around one moderate flare (factor $\gtrsim 2$ ) occurring daily on our sample CTTS. In addition to obvious flare events, variability is present also on smaller time scales and lower amplitude variations throughout the observations. 

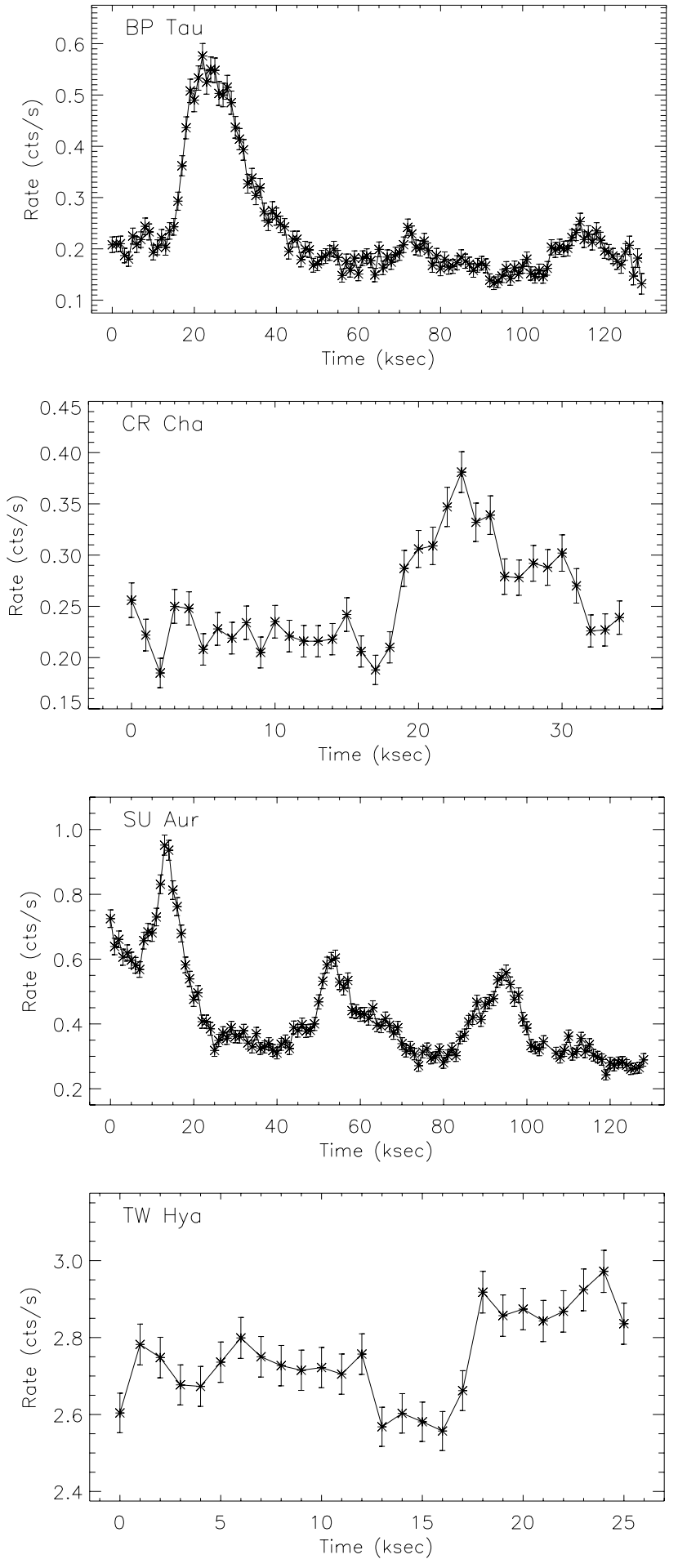

Fig. 1. Light curves and corresponding hardness ratio of the sample CTTS, PN (SU Aur-MOS) data with 1 ks binning.

\subsubsection{X-ray hardness}

The light curves in connection with spectral hardness can be used to identify the origin of the variability. While in typical stellar coronal flares the emission measure of predominantly hot plasma and hence the hardness of the spectra is increased, in a pure accretion spectrum no spectral changes should

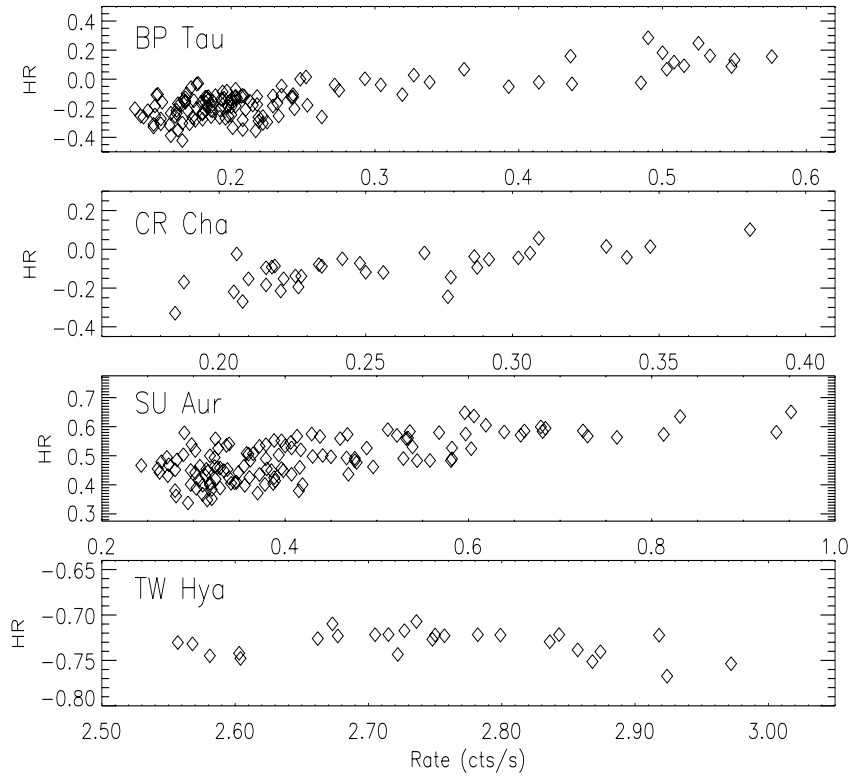

Fig. 2. Hardness ratio vs. count rate of the sample CTTS, PN (SU Aur-MOS) data with 1 ks binning.

accompany the brightening, since the plasma temperature only depends on the infall velocity and not the accretion rate. If, in addition, a coronal contribution with a temperature higher than that produced by accretion is present, a slight spectral softening should be observed. We calculate a hardness ratio for each light curve time bin and in Fig. 2 we show the hardness ratio vs. count rate for our sample CTTS. The hardness ratio is here defined as $\mathrm{HR}=\mathrm{H}-\mathrm{S} / \mathrm{H}+\mathrm{S}$ with the soft band covering the energy range $0.2-1.0 \mathrm{keV}$ and the hard band $1.0-10.0 \mathrm{keV}$. Errors are small compared to the observed shifts in hardness ratio. A clear correlation of X-ray brightness with spectral hardness is present for BP Tau, CR Cha and SU Aur, a behaviour typical of stellar flares, suggesting a coronal origin of the variability. No strong correlation is found for variations on TW Hya, which are, however, quite small. An anti-correlation appears to be present for the larger variations, as expected for brightness changes due to an increase in accretion rate. This is reflected in the slope of the linear regression curves. It is positive for BP Tau, CR Cha and SU Aur, but slightly negative for TW Hya.

To quantify the spectral changes that the CTTS are undergoing during phases of increasing emission in more detail, we use their light curves to define "high" states for BP Tau (15-37 ks), CR Cha (18.5-31.5 ks), SU Aur (0-24, 47-69, 83-101 ks) and TW Hya (>19 ks), while the remaining time intervals are considered as quasi-quiescence. An individual spectral analysis of both states of activity is presented in Sect. 4.2.3.

\subsection{Spectral analysis}

The spectral analysis and its results are presented as follows. First we use the medium resolution EPIC spectra in combination with the high resolution RGS data to obtain general spectral properties like temperatures, emission measures and elemental abundances of the X-ray emitting plasma and constrain the strength of absorption present for our targets. From 


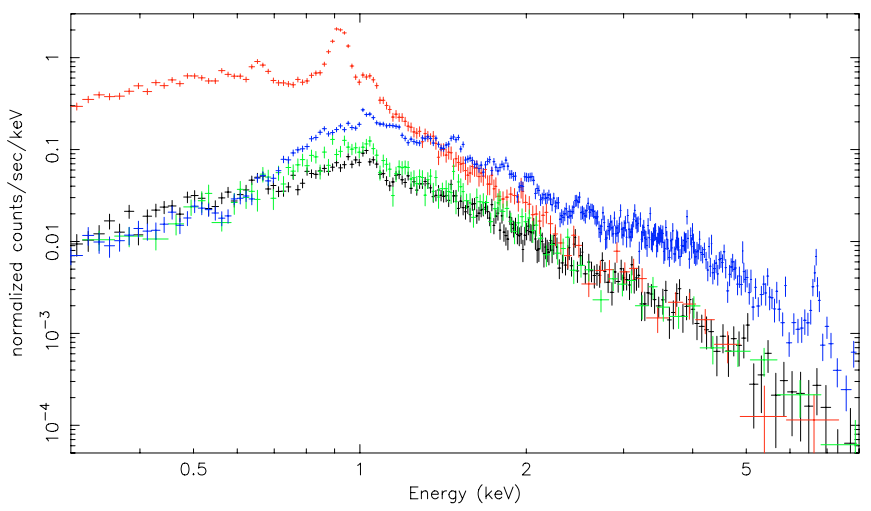

Fig. 3. Spectra of our sample CTTS for the total observation as observed with the MOS1 detector: BP Tau (black), CR Cha (green), SU Aur (blue) and TW Hya (red); bottom to top of the $1.0 \mathrm{keV}$ peak. Colour figure in electronic version.

the RGS spectra we investigate the resolved He-like triplet of O VII to determine the densities of the emitting plasma and/or strength of the surrounding UV field. Subsequently we investigate spectral changes between the low and the high state of our sample CTTS.

\subsubsection{The global spectra}

The spectra of all our sample CTTS as observed with MOS1 are shown in Fig. 3, which demonstrates major differences between individual stars. While the spectra of BP Tau and CR Cha are comparable, the spectrum of SU Aur indicates the presence of large amounts of extremely hot plasma noticeable, e.g. in the very strong Fe XXV line complex at $6.7 \mathrm{keV}$. On the other hand, the X-ray spectrum of TW Hya is much softer, suggesting a more dominant accretion component. The observed spectra are also subject to absorption, affecting primarily the energy range below $1.0 \mathrm{keV}$. In these spectra the low energy slope mainly reflects the strength of the absorption, while the high energy slope traces temperature and amount of hot coronal and flaring plasma. Inspection of the two slopes indicates that the absorption is weakest for TW Hya, moderate for BP Tau and strongest for CR Cha and SU Aur, while the coronal component is strongest and hottest for SU Aur, followed by BP Tau and CR Cha and much weaker and cooler for TW Hya.

To quantify the physical properties of the X-ray emitting plasma we fitted the spectra of the different instruments with multi-temperature spectral models as described in Sect. 3. The results of the fitting procedure are presented in Table 3. Note again the interdependence of several fitting parameter, especially absorption, cool emission measure and corresponding abundances. However, general trends on abundances and their ratios as well as the overall shape of the emission measure distribution and their changes, which indicate the production mechanism of the X-rays, are very stable.

While spectral modelling must consider absorption, the physically most interesting quantity is the emitted, i.e. dereddened, X-ray luminosity of our targets, which is calculated in the $0.2-10$. keV band using MOS data (see Table 3). We find that despite their very different spectral properties, all
Table 3. Spectral results, units are $N_{\mathrm{H}}$ in $10^{21} \mathrm{~cm}^{-2}, k T$ in keV EM in $10^{52} \mathrm{~cm}^{-3}$ and $L_{\mathrm{X}}$ in $10^{30} \mathrm{erg} \mathrm{s}^{-1}$.

\begin{tabular}{lcccc}
\hline \hline Par. & BP Tau & CR Cha & SU Aur & TW Hya \\
\hline$N_{\mathrm{H}}$ & $1.5_{-0.1}^{+0.1}$ & $2.7_{-0.3}^{+0.4}$ & $3.1_{-0.1}^{+0.1}$ & $0.35_{-0.05}^{+0.04}$ \\
$\mathrm{Fe}$ & $0.28_{-0.04}^{+0.04}$ & $0.45_{-0.07}^{+0.08}$ & $0.79_{-0.05}^{+0.05}$ & $0.26_{-0.02}^{+0.02}$ \\
$\mathrm{Si}$ & $0.14_{-0.08}^{+0.08}$ & $0.23_{-0.09}^{+0.10}$ & $0.60_{-0.08}^{+0.08}$ & $0.13_{-0.06}^{+0.05}$ \\
$\mathrm{O}$ & $0.62_{-0.06}^{+0.08}$ & $0.53_{-0.14}^{+0.18}$ & $0.39_{-0.11}^{+0.12}$ & $0.23_{-0.02}^{+0.02}$ \\
$\mathrm{Ne}$ & $1.47_{-0.14}^{+0.16}$ & $1.02_{-0.17}^{+0.19}$ & $1.04_{-0.14}^{+0.15}$ & $1.81_{-0.05}^{+0.05}$ \\
$k T 1$ & $0.20_{-0.01}^{+0.01}$ & $0.17_{-0.03}^{+0.03}$ & $0.68_{-0.02}^{+0.03}$ & $0.25_{-0.01}^{+0.01}$ \\
$k T 2$ & $0.63_{-0.03}^{+0.03}$ & $0.66_{-0.02}^{+0.01}$ & $1.61_{-0.10}^{+0.07}$ & $0.69_{-0.02}^{+0.06}$ \\
$k T 3$ & $2.17_{-0.09}^{+0.09}$ & $1.92_{-0.10}^{+0.06}$ & $4.82_{-0.84}^{+1.02}$ & $1.34_{-0.05}^{+0.03}$ \\
EM1 & $3.48_{-0.69}^{+0.89}$ & $4.96_{-2.49}^{+4.05}$ & $11.66_{-0.86}^{+0.87}$ & $19.16_{-0.82}^{+0.74}$ \\
EM2 & $5.28_{-0.56}^{+0.64}$ & $10.67_{-1.53}^{+1.60}$ & $25.27_{-3.96}^{+2.87}$ & $1.23_{-0.84}^{+0.54}$ \\
EM3 & $10.30_{-0.29}^{+0.43}$ & $9.27_{-0.54}^{+0.73}$ & $15.79_{-2.59}^{+4.02}$ & $3.12_{-0.51}^{+0.52}$ \\
\hline$\chi^{2}$ (d.o.f.) & $0.97(1281)$ & $1.20(700)$ & $1.20(1027)$ & $1.57(878)$ \\
\hline$L_{\mathrm{X}}$ obs. & 1.3 & 1.4 & 4.5 & 1.5 \\
$L_{\mathrm{X}}$ emit. & 2.3 & 3.1 & 8.1 & 2.0 \\
\hline
\end{tabular}

targets have an X-ray luminosity of the same order of magnitude. The hottest and most massive star SU Aur is also X-ray brightest target, followed by CR Cha, BP Tau and TW Hya with comparable X-ray luminosities. CTTS as a class are known strong X-ray emitter, but our sample stars are about a magnitude brighter than the X-ray luminosity of an average CTTS, as determined from RASS data with a mean $\log L_{\mathrm{X}}$ of 29.1 (Chamaeleon) and 29.4 (Taurus) in Neuhäuser et al. (1995), Feigelson et al. (1993); this is of course no surprise since these CTTS were selected for grating observations.

Neglecting interstellar contribution, the strength of absorption depends on the amount of circumstellar material in the disk and the inclination angle of the target. The results of our spectral modelling confirm that absorption is lowest for TW Hya. Our value for the interstellar column density $N_{\mathrm{H}}$ is slightly higher than the one used by Stelzer \& Schmitt (2004), who fixed $N_{\mathrm{H}}$ at $2 \times 10^{20} \mathrm{~cm}^{-2}$. No values are given for the Chandra data in Kastner et al. (2002), but the authors refer to previous work (Kastner et al. 1999) where values determined from ROSAT PSPC $\left(0.5 \times 10^{21} \mathrm{~cm}^{-2}\right)$ and higher ones from ASCA SIS $\left(2.9 \times 10^{21} \mathrm{~cm}^{-2}\right)$ with a consequently larger emission measure. These differences were attributed to temporal changes in the circumstellar environment, but the poorer spectral resolution and sensitivity of the used instruments, the strong interdependence between $N_{\mathrm{H}}$ and EM, that is also present in models from the individual instruments operated simultaneously onboard XMM-Newton, suggests that limited spectral resolution of the instruments combined with uncertainties in the code used for modelling the spectra is a possible explanation for those discrepancies. Recently Herczeg et al. (2004) analysed the Ly $\alpha$ profile of TW Hya as measured by HST/STIS and put a stringent upper limit on the hydrogen column density with $N_{\mathrm{H}} \leq 5 \times 10^{19} \mathrm{~cm}^{-2}$. We therefore adopted this value and additionally fitted the spectra with the reduced absorption value, which results in a comparable model, but with accordingly lower emission measure at cool temperatures. 
We find intermediate values of the interstellar column density $N_{\mathrm{H}}$ for BP Tau, which are in good agreement with optical measurements using the standard conversion between $N_{\mathrm{H}}$ and $A_{\mathrm{v}}$ viz. $N_{\mathrm{H}}=2 \times 10^{21} A_{\mathrm{v}} \mathrm{cm}^{-2}$; values taken from literature are in the range 1.0-2.0 $\times 10^{21} \mathrm{~cm}^{-2}$ (Gullbring et al. 1998; Hartigan et al. 1995). Even stronger absorption is found for CR Cha, also in good agreement with optical measurements, with a literature value of $2.7 \times 10^{21} \mathrm{~cm}^{-2}$ (Gauvin \& Strom 1992). The strongest absorption is found for SU Aur, here the large inclination angle results in a strong and probably also variable absorption. Optical and NIR measurements give a range of values ranging from no absorption up to $3 \times$ $10^{21} \mathrm{~cm}^{-2}$ (DeWarf et al. 2003). While commonly a lower $N_{\mathrm{H}}$ value is adopted, our result is comparable with the larger values and a much weaker absorption significantly worsens the quality of the fit.

The emission measure distributions (EMD) of our sample CTTS differ significantly among the sample stars. The hottest EMD is clearly present in SU Aur, with significant amounts of plasma at temperatures around $50 \mathrm{MK}$ detected. Due to the very strong absorption no definite conclusions about a possibly existing cool temperature part of the EMD can be drawn. Since SU Aur is of average age in our sample and the major difference to the other stars is its spectral type, an explanation for its outstanding properties might be a dependence of the evolutionary time scale on spectral type, i.e. stellar mass. The EMDs of BP Tau and CR Cha are similar, with the temperatures of BP Tau being slightly higher and a larger fraction of plasma residing in the hottest component; plasma at temperatures around $20-25 \mathrm{MK}$ must be present in those stars. The picture is very different for TW Hya, where the cool component with temperatures around $3 \mathrm{MK}$ clearly dominates the EMD. Plasma at medium temperatures with 5-10 MK appears to be nearly absent, while in the hot component plasma temperatures around $15 \mathrm{MK}$ are reached; however, this component is cooler and significantly weaker than in the other objects. We emphasise that this finding is independent of the used value of $N_{\mathrm{H}}$.

As far as abundances are concerned, one property is common to all CTTS analysed: Most abundances are subsolar, sometimes at considerable level, while neon is commonly found at solar abundance or even significantly enhanced. The noble gas neon is more abundant compared to iron, oxygen and silicon for all targets, and in the most extreme case TW Hya these ratios are of the order of 10 (see Table 3). Iron and silicon are less abundant than oxygen in BP Tau and CR Cha, while all three elements are strongly depleted in TW Hya. The very active star SU Aur appears to be an exception, with only oxygen apparently being more strongly depleted, but here very strong absorption affects the results based on cool lines. These metal anomalies were interpreted for TW Hya by Stelzer \& Schmitt (2004) through a depletion of grain forming element in the accreted material, consistent with the low observed IR excess and also plausible when considering the age of TW Hya. Drake et al. (2005) argue, that for TW Hya coagulation of grains into larger bodies finally withdraws these elements from the accretion process, while for the younger BP Tau dust and grains at corotation radius $\left(R_{\mathrm{co}}=\left(\frac{G M}{\omega^{2}}\right)^{1 / 3}\right)$ will sublimate and rereleased into the accretion process. The coagulation of significant amounts of material into centimeter sized particles in the disk around TW Hya is supported by the radiospectrum at centimeter wavelength as observed with the VLA (Wilner et al. 2005).

However, stars without accretion but with an active corona also show a distinct abundance pattern, commonly known as IFIP effect, i.e. an enhancement of elements with a high first ionisation potential. While iron and silicon are low FIP elements, oxygen is an intermediate and neon a high FIP element. The IFIP-effect is observed for BP Tau and CR Cha, thus pointing towards the typical abundance pattern for coronal plasma. Moreover, Argiroffi et al. (2005) analyse the spectra of TWA5, a young multiple system dominated by a $\mathrm{M}$ dwarf and also located in the TW Hya association. These object is classified as CTTS (via $\mathrm{H} \alpha$ ), but is probably a WTTS since there is no evidence for a disk in the IR, but it also shows a high neon to iron ratio similar to TW Hya with spectral properties very much reminiscent of active stars. While environmental conditions may be invoked to explain the abundance anomalies, Robrade \& Schmitt (2005) showed that a high neon to iron ratio is common for active $\mathrm{M}$ dwarfs, with most extreme ratios found in very young and therefore more active stars. We further point out that our CTTS belong to different star formation regions and their X-ray emission is predominantly generated by different processes, yet no significant difference in the abundance pattern was found. Therefore it appears reasonable to attribute the observed abundance anomalies in the sample CTTS to a combined action of metal depletion of the accreted matter via grain forming and the coronal IFIP effect. Grain forming is only important for the accretion plasma of older and more evolved objects, while the IFIP effect is present in plasma generated in active coronae.

\subsubsection{The O VIII and O VII lines}

We used the CORA line fitting program to determine the strengths of the resonance, intercombination and forbidden lines in the He-like triplet of O VII $(21.6,21.8,22.1 \AA)$ and the Ly $\alpha$ line of O VIII at $18.97 \AA$ as measured with RGS1. The spectra of the sample CTTS covering this wavelength-region are shown in Fig. 4, the measured line counts of the O VII triplet and the derived densities are given in Table 4 . We used the relation $f / i=\frac{R_{0}}{1+\phi / \phi_{\mathrm{c}}+n e / N_{\mathrm{c}}}$ with $f$ and $i$ being the line intensities in the forbidden and intercombination line, $R_{0}$ the low density limit of the line ratio with a adopted value of $3.95, N_{\mathrm{c}}$ the critical density and $\phi / \phi_{c}$ the radiation term, which is neglected in our calculations. Values used in the calculations were taken from (Pradhan \& Shull 1981); we caution that the presence of strong radiation fields would lead us to overestimate plasma densities. The O VII triplet traces the cool plasma around $2 \mathrm{MK}$, is essentially free of stronger blends and therefore well suited to investigate possible accretion scenarios, while O VIII traces slightly hotter plasma around 3-5 MK.

The analysis of the oxygen triplet of BP Tau and its implications are presented by Schmitt et al. (2005), results obtained for TW Hya are presented in Stelzer \& Schmitt (2004). Our analysis here is - technically - slightly different, but we arrive at the same results as found by Stelzer \& Schmitt (2004). The 

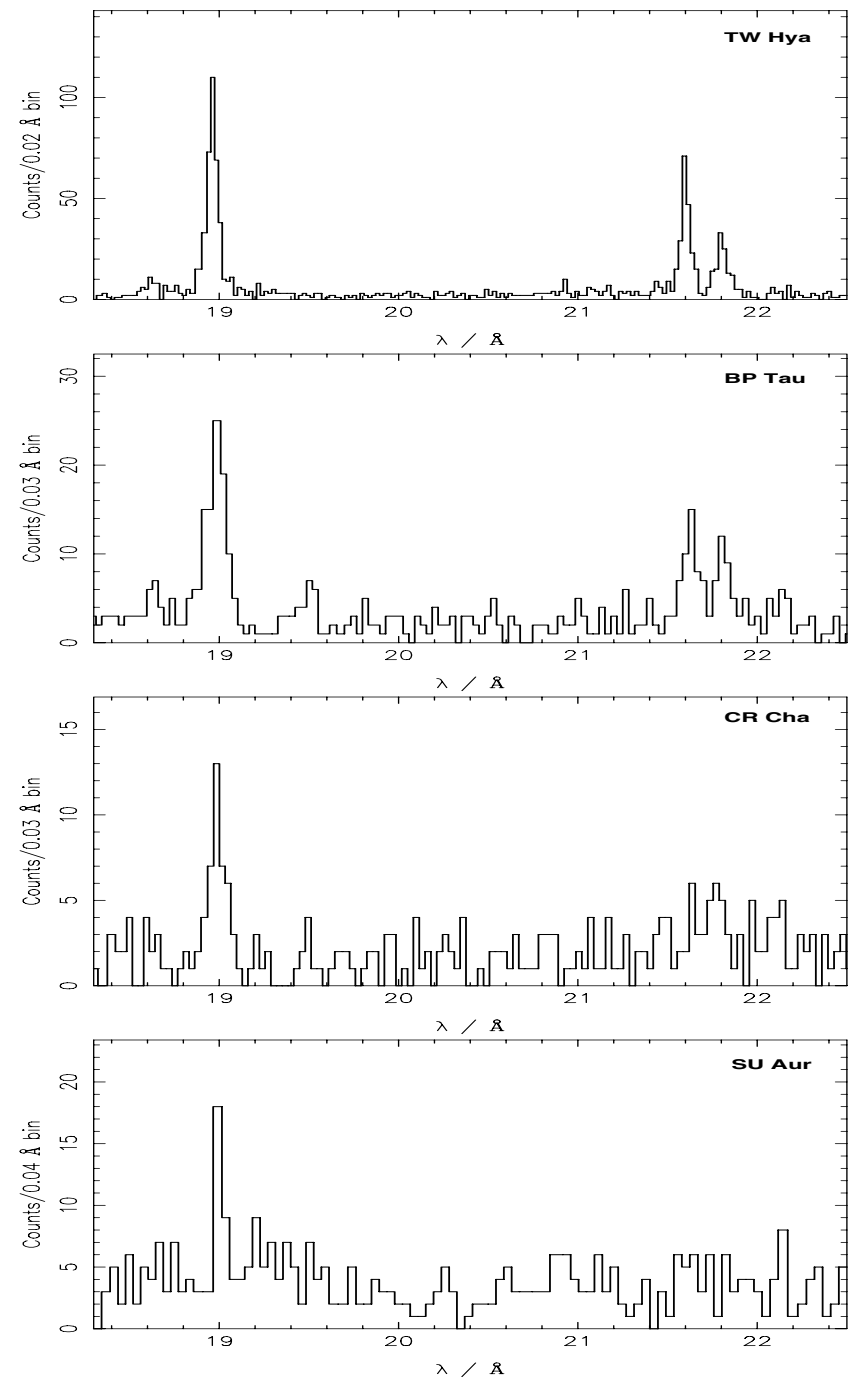

Fig. 4. Spectra of the sample CTTS covering the O VIII line and the O VII triplet as measured with the RGS1 detector. Absorption increases from the top to bottom.

data of CR Cha, which unfortunately has only moderate S/N ratio and larger absorption, is analysed here for the first time. The O VIII line is here clearly detected, the same applies to the O VII triplet, which, however, looks quite peculiar. Resonance and forbidden lines have about the same strength, and the intercombination line is actually the strongest line in the triplet. Because of the poor SNR the errors in the line count measurements are quite substantial; also the $g$-ratio $(f+i) / r$ is found to be $2.9 \pm 2.2$, but is because of its large error actually consistent with unity as theoretically expected. The observed $f / i$ ratio is well below unity despite its considerable measurement error, as observed for TW Hya and BP Tau.

In SU Aur the O VII triplet is absent, ruling out any quantitative analysis. We attribute the absence of O VII to the strong (circumstellar) absorption and the presence of extremely hot plasma, which increases the continuum emission at these wavelengths. Some weak emission may actually be present at the position of the oxygen triplet, but neither the intercombination nor forbidden line are clearly detected. The O VIII Ly $\alpha$ line is however clearly present and attenuation increases only by
Table 4. Measured RGS1 line counts of the O VIII and O VII-triplet ( $r$, $i, f$ ) lines and calculated densities, taking into account the effective areas at the respective wavelengths.

\begin{tabular}{lcccc}
\hline \hline Par. & BP Tau & CR Cha & SU Aur & TW Hya \\
\hline O VIII & $94.5 \pm 11.4$ & $36.6 \pm 7.1$ & $33.1 \pm 7.5$ & $453.0 \pm 23.8$ \\
O VII $r$ & $44.9 \pm 8.4$ & $7.5 \pm 4.9$ & - & $234.9 \pm 17.3$ \\
O VII $i$ & $34.5 \pm 7.6$ & $13.5 \pm 5.6$ & - & $113.5 \pm 12.9$ \\
O VII $f$ & $11.6 \pm 5.3$ & $8.7 \pm .8$ & - & $6.1 \pm 5.1$ \\
$f / i($ obs. $)$ & $0.34 \pm 0.17$ & $0.64 \pm 0.44$ & - & $0.054 \pm 0.045$ \\
\hline$n_{\mathrm{e}}\left(10^{11} \mathrm{~cm}^{-3}\right)$ & $3.2_{-1.2}^{+3.5}$ & $1.6_{-0.8}^{+4.2}$ & - & $21.1_{-9.7}^{+107}$ \\
\hline
\end{tabular}

a factor of roughly two between the two wavelengths. A strong O VII triplet should therefore be detected in the spectra, even with SU Aur's strong absorption. This indicates, that large amounts of cool plasma do not exist on SU Aur.

Comparing the ratio of $\mathrm{O}$ VIII to the $\mathrm{O}$ VII line counts, we find the largest (observed) ratio for TW Hya. However, absorption attenuates the $\mathrm{O}$ VII lines more strongly than the $\mathrm{O}$ VIII line and using our determined values for $N_{\mathrm{H}}$, in the unabsorbed ratio the inverse trend is observed. While typical active coronae, as well as all other sample CTTS, show a rise of the EMD in this temperature regime, we find that in the accretion dominated spectrum of TW Hya the bulk of plasma has temperatures around 2.5-3 MK, consistent with the results of the global fitting.

Investigating the $\mathrm{O}$ VII triplets of our sample CTTS, we find that derived densities differ from those of cool main sequence stars. While typical coronal plasma is compatible with and for very active stars no more than an order of magnitude above the low density limit, i.e. $f / i \sim 2-4$ and $\log n_{\mathrm{e}} \sim 9-10$ (Ness et al. 2002), all CTTS with detected oxygen triplets deviate strongly from the low density limit. BP Tau and CR Cha show a density about two orders of magnitude lower, for TW Hya it is even three orders of magnitude lower, suggesting extremely high densities for the cool plasma of the CTTS. Alternatively, strong UV radiation fields would explain the measured line ratios, but these fields cannot be produced by the stellar photospheres of the underlying cool stars, therefore the radiation fields would have to be attributed to an additional hotter component, i.e. an accretion shock.

Thus the derived densities may be considered as an upper limit, but we point out that even in the case of UV radiation contributing to the low $f / i$-ratio accretion must occur, since also a strong UV radiation points to the presence of an accretion-induced shocks. Furthermore, the coronal plasma is likely to additionally contribute to the flux in the $\mathrm{O}$ VII-triplet. It was shown in Schmitt et al. (2005) that the $f / i$-ratio for BP Tau is even lower, when only the quasi-quiescent state is considered in the analysis. This is plausible, because the $f / i$-ratio from the coronal contribution is expected to be near the low density limit and therefore the densities in the accretion spot are underestimated in this calculation and also appear as an lower limit. Since the size of the accretion spots or filling factors as well as the amount of coronal plasma contributing at low temperatures are not known precisely, we just 
note that there are two competing effects on the derived densities present, but they are not calling the accretion scenario in question. While the poor data quality for CR Cha prevents a more detailed discussion as for BP Tau, the derived $f / i$-ratio is also significantly lower than for coronal sources and the derived plasma density is in the same order of magnitude as it is for BP Tau. The derived results for CR Cha fit into the picture and support the accretion scenario for CTTS as a class. Therefore we conclude that the cool plasma of TW Hya is strongly dominated by accretion, while in the cases of BP Tau and CR Cha and possibly also of SU Aur accretion is present, but with additional cool coronal plasma. However, the emission measure of this component is hard to constrain given the SNR of our data. These findings agree well with the conclusions drawn in the previous section.

\subsubsection{Spectral changes}

To investigate the spectral changes between the phases of different X-ray brightness, we use the more sensitive EPIC data, separated in low and high states as defined in Sect. 4.1 and utilise the previously derived best fit models. A comparison of the PN spectra of BP Tau and TW Hya during high and low state is shown in Fig. 5, where the high state represents the strong flare on BP Tau (factor 2.4) and the period of slightly enhanced emission of TW Hya (factor 1.1). The differences between the spectra of the high and low state are nearly reverse for the two CTTS. While for BP Tau the spectrum hardens and the most significant changes are seen at higher energies, pointing to a coronal origin of the changes, the TW Hya spectra are identical at higher energies and only at lower energies the emission is enhanced, pointing to a higher accretion rate. The changes are more pronounced for BP Tau due to the much higher increase in X-ray brightness.

No significant changes in the elemental abundances were detected between high and low state, therefore we fixed strength of absorption and abundances at those values that were derived for the total observation and remodelled the EMD for the selected time intervals. If we find - using temperature as a free parameter for each state - no significant difference in the temperature components, it is tied for both sets of data.

The results of the individual modelling of the low and high states for our sample CTTS are summarised in Table 5. Very different effects on the derived temperature structures are found and also the largest increase in emission measure is found in different temperature components for our CTTS. The rise in temperature and the presence of a larger amount of additional hot plasma with temperatures around $30 \mathrm{MK}$ during the high state reflects the coronal origin of the increased X-ray brightness for BP Tau. Flaring affects also the emission measure at moderate temperatures as represented by the increased emission measure and a shift to higher temperatures, which are in the range of 6-8 MK. A more moderate increase of temperatures and additional plasma at moderate temperatures but again predominantly in the hot component is found for CR Cha. This also points to a coronal origin of the increased brightness and is consistent with the observed more moderate flare. Also for

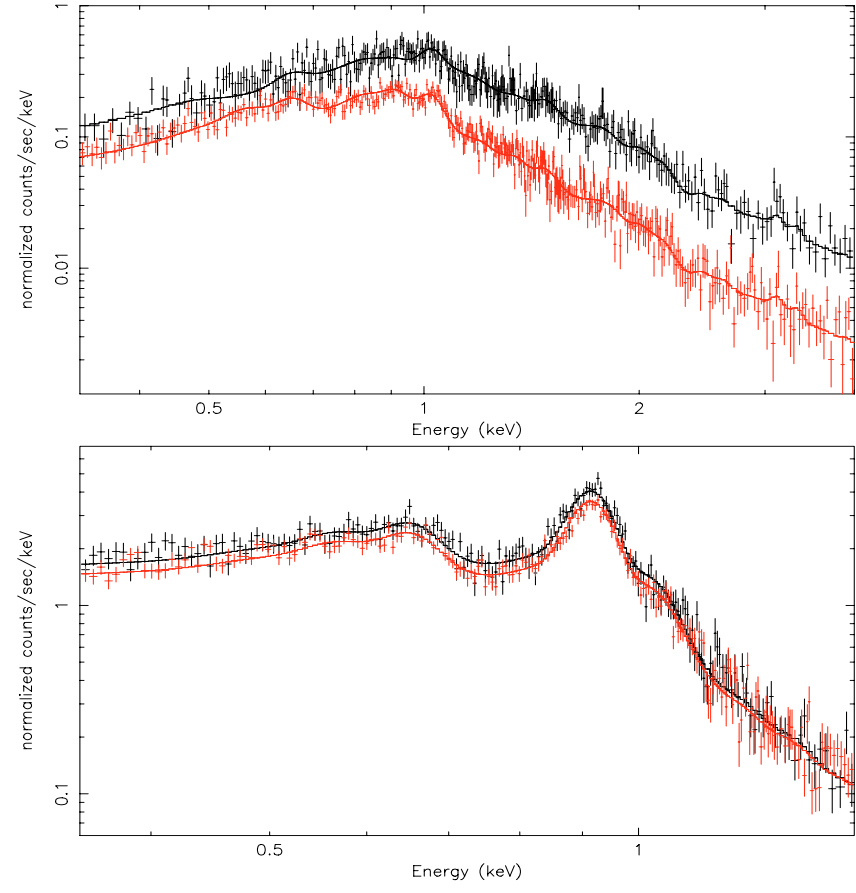

Fig. 5. Spectra of BP Tau (top) and TW Hya (bottom) during high (black) and low (red) state with model fits.

Table 5. EMD of our sample CTTS in low (left) and high (right) state as derived from PN data (MOS for SU Aur), $N_{\mathrm{H}}$ in $10^{21} \mathrm{~cm}^{-2}$, $k T$ in $\mathrm{keV}$ and $\mathrm{EM}$ in $10^{52} \mathrm{~cm}^{-3}$.

\begin{tabular}{|c|c|c|c|c|}
\hline Par. & \multicolumn{2}{|c|}{ BP Tau } & \multicolumn{2}{|c|}{ CR Cha } \\
\hline$N_{\mathrm{H}}$ & \multicolumn{2}{|c|}{1.5} & \multicolumn{2}{|c|}{2.6} \\
\hline$k T 1$ & \multicolumn{2}{|c|}{$0.18_{-0.02}^{+0.02}$} & \multicolumn{2}{|c|}{$0.19_{-0.04}^{+0.06}$} \\
\hline$k T 2$ & $0.50_{-0.05}^{+0.07}$ & $0.67_{-0.07}^{+0.07}$ & \multicolumn{2}{|c|}{$0.65_{-0.03}^{+0.03}$} \\
\hline kT3 & $2.12_{-0.14}^{+0.15}$ & $2.71_{-0.14}^{+0.07}$ & $1.71_{-0.19}^{+0.20}$ & $1.99_{-0.14}^{+0.23}$ \\
\hline EM1 & $4.5_{-0.5}^{+0.5}$ & $2.9_{-1.0}^{+1.0}$ & $4.5_{-1.2}^{+1.5}$ & $6.3_{-2.3}^{+2.9}$ \\
\hline EM2 & $5.9_{-0.5}^{+0.4}$ & $9.8_{-1.2}^{+0.9}$ & $10.8_{-1.7}^{+1.5}$ & $11.7_{-1.9}^{+1.4}$ \\
\hline EM3 & $6.8_{-0.4}^{+0.5}$ & $23.9_{-1.7}^{+1.4}$ & $7.7_{-0.9}^{+0.9}$ & $13.3_{-1.4}^{+1.3}$ \\
\hline$\chi^{2}$ (d.o.f.) & \multicolumn{2}{|c|}{$0.89(778)$} & \multicolumn{2}{|c|}{$1.12(414)$} \\
\hline Par. & \multicolumn{2}{|c|}{ SU Aur } & \multicolumn{2}{|c|}{ TW Hya } \\
\hline$N_{\mathrm{H}}$ & \multicolumn{2}{|c|}{3.1} & \multicolumn{2}{|c|}{0.35} \\
\hline$k T 1$ & \multicolumn{2}{|c|}{$0.69_{-0.02}^{+0.03}$} & \multicolumn{2}{|c|}{$0.24_{-0.01}^{+0.01}$} \\
\hline$k T 2$ & \multicolumn{2}{|c|}{$1.62_{-0.07}^{+0.09}$} & \multicolumn{2}{|c|}{$0.60_{-0.06}^{+0.09}$} \\
\hline$k T 3$ & \multicolumn{2}{|c|}{$5.08_{-0.81}^{+1.27}$} & \multicolumn{2}{|c|}{$1.37_{-0.05}^{+0.11}$} \\
\hline EM1 & $10.8_{-0.5}^{+1.0}$ & $11.9_{-0.3}^{+1.1}$ & $21.5_{-0.6}^{+0.4}$ & $24.5_{-0.8}^{+0.4}$ \\
\hline EM2 & $21.2_{-2.1}^{+1.8}$ & $29.9_{-4.3}^{+4.1}$ & $2.3_{-0.4}^{+0.8}$ & $3.2_{-0.3}^{+1.1}$ \\
\hline EM3 & $6.9_{-1.9}^{+1.9}$ & $22.7_{-4.3}^{+4.4}$ & $2.8_{-0.6}^{+0.3}$ & $2.3_{-0.8}^{+0.5}$ \\
\hline$\chi^{2}$ (d.o.f.) & \multicolumn{2}{|c|}{$1.10(929)$} & \multicolumn{2}{|c|}{$1.27(496)$} \\
\hline
\end{tabular}

SU Aur coronal activity is the explanation for the increased X-ray brightness, with large amounts of additional hot plasma at temperatures of 20-50 MK. The flare plasma is extremely hot, but since large amounts of extremely hot plasma are already present during quasi-quiescence, no significant increase in temperature accompanies the brightening. The coolest detected component of SU Aur, which already has temperatures 
around $8 \mathrm{MK}$, is again not strongly affected by the flaring. In contrast, none of these signatures are connected with the flux increase for TW Hya. No differences in the temperature structure were found and the additional plasma is found mainly in the coolest temperature component, supporting the scenario of increased accretion. With 2.5-3 MK this component is much cooler than typical flare plasma temperatures, but fits to the temperatures as expected for plasma generated in an accretion spot.

\section{Summary and conclusions}

We have presented the first comparative study using high and medium resolution X-ray spectra of classical T Tauri stars observed with the new generation X-ray telescopes so far. The results derived from these data are complementary to the ones obtained from the large sample of COUP sources, since highresolution spectroscopy and low-energy sensitivity additionally permits the investigation of the cool plasma components.

Using the XMM-Newton observations of BP Tau, CR Cha, SU Aur and TW Hya we determined X-ray properties of accreting young pre-main sequence stars. Two different mechanisms are likely to contribute to the production of X-ray emission in those objects, first coronal, i.e. magnetic activity, and second, magnetically funneled accretion. We investigate variability, global spectra and density sensitive lines to check for the presence and relative contribution of these two mechanisms for the individual objects. For this purpose we utilise the emission measure distribution and its changes, specific abundance pattern and density diagnostics. Interpreting very high densities as indicator for accretion shocks and a high temperature component as indicator for coronal activity, we derive the following conclusions which are likewise supported by the analysis of abundance analysis and spectral variability. In all targets where the $\mathrm{O}$ VII-triplet is observable density analysis leads to higher densities than observed in any pure coronal source. Additionally, a high temperature component is present in all targets. We therefore argue that both X-ray generating processes are present in our sample CTTS, but at very different levels and importance in individual objects.

The X-ray emission of BP Tau is overall dominated by coronal activity and a stronger flare of again probably coronal origin is observed. Even in quasi-quiescent phases the emission measure distribution is dominated by medium and hot temperature plasma whereof significant amounts are present at temperatures around $20 \mathrm{MK}$. However, the analysis of density sensitive lines confirms that accretion is also present and actually dominates the cool plasma with temperatures of a few MK. CR Cha is similar to BP Tau but more strongly absorbed and the coronal plasma temperatures are slightly lower. It also appears to be dominated by the coronal contribution, likewise there is evidence for high density plasma at cool temperatures. SU Aur is the by far brightest and with temperatures of at least up to $50 \mathrm{MK}$ hottest X-ray source in our sample. A powerful and active corona is the essential contributor to its X-ray spectrum and frequent flaring is observed. Unfortunately the very strong absorption prevents definite conclusions about the cooler plasma component. TW Hya is the other hand strongly dominated by accretion, but an additional coronal component is clearly detected. It is the prototype and still the outstanding and only example of an accretion dominated star. Most of its plasma is at cool temperatures typical for accretion spots and it exhibits by far the lowest O VII $f / i$ ratio. Also the highest neon to oxygen ratio, which may be interpreted via depletion of grain forming element, is found for TW Hya. This is plausible since TW Hya is the oldest and most evolved object in our sample. In the other sample CTTS neon is also enhanced, but the abundance pattern is more reminiscent of the inverse FIP effect found in active stars.

Considering the global picture of stellar evolution towards the main sequence, we find that magnetic processes play a major role in high energy phenomena in all our sample CTTS. While the specific stellar evolution and its timescale might depend on e.g. spectral type, the generation of X-rays via accretion and coronal activity is apparently a common feature of CTTS in general.

Acknowledgements. This work is based on observations obtained with XMM-Newton, an ESA science mission with instruments and contributions directly funded by ESA Member States and the USA (NASA). This research has made use of the SIMBAD database, operated at CDS, Strasbourg, France. (http://simbad.u-strasbg.fr) J.R. acknowledges support from DLR under 50OR0105.

\section{References}

Anders, E., \& Grevesse, N. 1989, Geo. Cosmochim. Acta, 53, 197

Argiroffi, C., Maggio, A., Peres, G., et al. 2005, A\&A, 439, 1149

Arnaud, K. A. 1996, ASP Conf. Ser., 101, 17

Asplund, M. 2005, ASP Conf. Ser., 336, 25

Calvet, N., \& Gullbring, E. 1998, ApJ, 509, 802

DeWarf, L. E., Sepinsky, J. F., Guinan, E. F., et al. 2003, ApJ, 590, 357

Drake, J. J., Testa, P., \& Hartmann, L. 2005, ApJ, 627, L149

Ehle, M., Breitfellner, M., Gonzales Riestra, M., et al. 2003, XMM-Newton User's Handbook

Favata, F., Flaccomio, E., Reale, F., et al. 2005, ApJS, 160, 469

Feigelson, E. D., \& DeCampli, W. M. 1981, ApJ, 243, L89

Feigelson, E. D., \& Kriss, G. A. 1989, ApJ, 338, 262

Feigelson, E. D., Casanova, S., Montmerle, T., \& Guibert, J. 1993, ApJ, 416, 623

Feigelson, E. D., \& Montmerle, T. 1999, ARA\&A, 37, 363

Feigelson, E. D., Gaffney, J. A., Garmire, G., et al. 2003, ApJ, 584, 911

Grevesse, N., \& Sauval, A. J. 1998, Space Sci. Rev., 85, 161

Gauvin, L. S., \& Strom, K. M. 1992, ApJ, 385, 217

Güedel, M., Skinner, S. L., Briggs, K. R., et al. 2005, ApJ, 626, L53

Gullbring, E., Barwig, H., \& Schmitt, J. H. M. M. 1997, A\&A, 324, 155

Gullbring, E., Hartmann, L., Briceno, C., \& Calvet, N. 1998, ApJ, 492, 323

Hartigan, P., Edwards, S., \& Ghandour, L. 1995, ApJ, 452, 736

Herczeg, G. J., Wood, B. E., Linsky, J. L., et al. 2004, ApJ, 607, 369

Kastner, J. H., Huenemoerder, D. P., Schulz, N. S., et al. 1999, ApJ, 525,837 
Kastner, J. H., Huenemoerder, D. P., Schulz, N. S., et al. 2002, ApJ, 567,434

Kirsch, M. G. F., Altieri, B., Chen, B., et al. 2004 [arXiv: astro-ph/0407257]

Loiseau, N., et al. 2004, User's Guide to XMM-Newton Science Analysis System Issue 3.1, ed. M. Ehle, \& A. M. T. Pollock

Makarov, V. V., \& Fabricius, C. 2001, A\&A, 368, 866

Meeus, G., Sterzik, M., Bouwman, J., \& Natta, A. 2003, A\&A, 409, L25

Muzerolle, J., Calvet, N., Hartmann, L., \& D’Alessio, P. 2003, ApJ, 597, L149

Natta, A., Meyer, M. R., \& Beckwith, S. V. W. 2000, ApJ, 534, 838

Ness, J.-U., Schmitt, J. H. M. M., Burwitz, V., et al. 2002, A\&A, 394, 911

Ness, J.-U., \& Wichmann, R. 2002, AN, 323, 129

Neuhäuser, R., Sterzik, M. F., Schmitt, J. H. M. M., et al. 1995, A\&A, 297, 391

Pallavicini, R., Franciosini, E., \& Randich, S. 2004, MSAI, 75, 434
Pradhan, A. K., \& Shull, J. M. 1981, ApJ, 249, 821

Preibisch, T., Kim, Y.-C., Favata, F., et al. 2005, ApJS, 160, 401

Reipurth, B., Pedrosa, A., \& Lago, M. T. V. T. 1996, A\&AS, 120, 229

Robrade, J., \& Schmitt, J. H. M. M. 2005, A\&A, 435, 1073

Schmitt, J. H. M. M., Robrade, J., Ness, J.-U., et al. 2005, A\&A, 432, L35

Shu, F., Najita, J., Ostriker, E., et al. 1994, ApJ, 429, 781

Smith, R. K., Brickhouse, N. S., Liedahl, D. A., \& Raymond, J. C. 2001, ASP Conf. Ser., 247, 161

Stelzer, B., \& Neuhäuser, R. 2001, A\&A, 377, 538

Stelzer, B., \& Schmitt, J. H. M. M. 2004, A\&A, 418, 687

Walter, F. M., \& Kuhi, L. V. 1981, ApJ, 250, 254

Wichmann, R., Bastian, U., Krautter, J., et al. 1998, MNRAS, 301, L39

Wilner, D. J., Alessio, P. D., Calvet, N., et al. 2005, ApJ, 626, L109

Zuckerman, B., Webb, R. A., Schwartz, M., \& Becklin, E. E. 2001, ApJ, 549, L233 